\title{
Gênero Candida - Fatores de virulência, Epidemiologia, Candidíase e Mecanismos de
}

\section{resistência}

\author{
Candida genus - Virulence factores, Epidemiology, Candidiasis and Resistance mechanisms \\ Género Candida - Factores de virulencia, Epidemiología, Candidiasis Y Mecanismos de resistência
}

Recebido: 25/03/2021 | Revisado: 03/04/2021 | Aceito: 08/04/2021 | Publicado: 17/04/2021

\author{
Wilma Raianny Vieira da Rocha \\ ORCID: https://orcid.org/0000-0001-8126-8153 \\ Universidade Federal de Pernambuco, Brasil \\ E-mail: wilmaraianny@gmail.com \\ Luanne Eugênia Nunes \\ ORCID: https://orcid.org/0000-0001-6524-0994 \\ Faculdade Nova Esperança de Mossoró, Brasil \\ E-mail: luanne_87@hotmail.com \\ Marina Luizy Rocha Neves \\ ORCID: https://orcid.org/0000-0002-7949-6605 \\ Universidade Federal de Pernambuco, Brasil \\ E-mail: marinaluizy@hotmail.com \\ Eulália Camelo Pessoa de Azevedo Ximenes \\ ORCID: https://orcid.org/0000-0002-4486-0374 \\ Universidade Federal de Pernambuco, Brasil \\ E-mail: eulaliaximenes@yahoo.com.br \\ Mônica Camelo Pessoa de Azevedo Albuquerque \\ ORCID: https://orcid.org/0000-0002-2882-6563 \\ Universidade Federal de Pernambuco, Brasil \\ E-mail: monica.aalbuquerque@ufpe.br
}

\begin{abstract}
Resumo
Gênero Candida é encontrado vivendo de forma simbionte na microbiota da mucosa reprodutiva e gastrointestinal de 50-70\% dos indivíduos saudáveis, em determinadas condições esses micro-organismos podem se tornar patogênicos, a ponto de se tornar a terceira causa das septicemias ao redor do mundo. Podem causar infecções superficiais, atingindo mucosas e pele, bem como infecções invasivas, em órgãos e tecidos profundos como intestino, pulmão e sangue. A espécie mais isolada em infecções causadas pelo gênero é a Candida albicans, porém é cada vez mais recorrente o isolamento de espécies de Candida não-albicans, como: C. glabrata, C. krusei, C. tropicalis e C. parapsilosis. Além disso, $C$. albicans e espécies de $C$. não-albicans vêm sendo cada vez mais relatadas pelo aparecimento de resistência aos antifúngicos de uso clínico, dificultando o tratamento de infecções causadas por estas cepas. Este trabalho teve como objetivo reunir informações atualizadas sobre o gênero Candida sobre fatores de virulência, epidemiologia, candidíase, tratamentos e mecanismos de resistência.
\end{abstract}

Palavras-chave: Candida albicans; $C$. não-albicans; Candidíase; Fatores de virulência; Mecanismos de resistência.

\begin{abstract}
The genus Candida is found symbiotically living in the microbiota of the reproductive and gastrointestinal mucosa of $50-70 \%$ of healthy individuals, under certain conditions these microorganisms can become pathogenic, to the point of becoming the third cause of septicemia worldwide. They can cause superficial infections, affecting mucous membranes and skin, as well as invasive infections, in organs and deep tissues such as intestines, lungs and blood. The most isolated species in infections caused by the genus is Candida albicans, however the isolation of species of Candida non-albicans, such as C. glabrata, C. krusei, C. tropicalis and C. parapsilosis is increasingly recurrent. In addition, $C$. albicans and $C$. non-albicans species have been increasingly reported due to the emergence of resistance to antifungal agents for clinical use, making it difficult to treat infections caused by these strains. This work aimed to gather updated information about the genus Candida on virulence factors, epidemiology, candidiasis, treatments and resistance mechanisms.
\end{abstract}

Keywords: Candida albicans; C. non-albicans; Candidiasis; Virulence factors; Resistance mechanisms.

\section{Resumen}

El género Candida se encuentra viviendo simbióticamente en la microbiota de la mucosa reproductiva y gastrointestinal de 50-70\% de individuos sanos, bajo ciertas condiciones estos microorganismos pueden volverse patógenos, hasta el punto de convertirse en la tercera causa de septicemia a nivel mundial . Pueden causar infecciones 
superficiales, afectando membranas mucosas y piel, así como infecciones invasivas, en órganos y tejidos profundos como intestinos, pulmones y sangre. La especie más aislada en infecciones causadas por el género es Candida albicans, sin embargo el aislamiento de especies de $C$. non-albicans, como $C$. glabrata, C. krusei, C. tropicalis y $C$. parapsilosis es cada vez más recurrente. Además, se han reportado cada vez más especies de $C$. albicans y $C$. nonalbicans debido a la aparición de resistencias a agentes antifúngicos para uso clínico, lo que dificulta el tratamiento de infecciones causadas por estas cepas. Este trabajo tuvo como objetivo recopilar información actualizada sobre el género Candida sobre factores de virulencia, epidemiología, candidiasis, tratamientos y mecanismos de resistencia.

Palabras clave: Candida albicans; . no-albicans; Candidiasis; Factores virulentos; Mecanismos de resistência.

\section{Introdução}

O gênero Candida apresenta estrutura celular característica dos seres vivos do Reino Fungi, que inclui a parede celular de quitina e a membrana citoplasmática fosfolipídica, constituída por proteínas, que agem como enzimas, e o ergosterol. Em condições ótimas de crescimento, com nutrientes específicos e temperatura ideal, crescem exponencialmente em forma de blastoconídios (Modrzewska \& Kurnatowski, 2013).

Os micro-organismos do gênero Candida são encontrados na microbiota da mucosa reprodutiva e gastrointestinal, vivendo de forma simbionte, em cerca de 50-70\% dos indivíduos saudáveis. Este gênero é formado por micro-organismos oportunista, que acometem, principalmente, pacientes imunodeprimidos, como também em tratamento com antimicrobianos de amplo espectro. Estas condições tornam esses micro-organismos importantes agentes causadores de infecção, como a Candidíase, que podem ser superficiais ou invasivas (Pappas, Lionakis, Arendrup, Ostrosky-Zeichner \& Kullberg, 2018).

Dos fungos leveduriformes responsáveis por causar doenças em humanos, as espécies pertencentes ao gênero Candida aparecem em destaque (Silva et al., 2012). C. albicans é uma espécie prevalente nas infecções causadas por este gênero. No entanto, o número de relatos de infecções causadas por espécies de Candida não-albicans, como C. glabrata é crescente. Os principais problemas destas espécies é a alta incidência de infecções em adultos associada a elevada mortalidade, decorrente do aumento de micro-organismos que apresentam resistência aos antifúngicos, com destaque para a classe dos azólicos e equinocandinas (Pfaller et al., 2010; Castanheira, Messer, Rhomberg, \& Pfaller, 2016). Para o tratamento de candidíases superficiais e invasivas os antifúngicos azóis são indicados nos casos de infecções causadas por leveduras sensíveis a estes medicamentos e que causam principalmente candidíase mucocutânea oral, vaginal e esofágica, além de candidíase na pele e nas unhas. Mas também são indicados para candidíase invasiva, para estes casos, principalmente o fluconazol, itraconazol, cetoconazol e voriconazol (Hani, Shivakumar, Vaghela, Osmani, \& Shrivastava, 2015).

Este artigo teve como objetivo realizar um levantamento bibliográfico com atualizações sobre o gênero Candida, destacando os fatores de virulência, epidemiologia, aspectos relacionados a candidíase e tratamento, bem como os mecanismos de resistência do gênero Candida aos antifúngicos disponíveis e que estão relacionados com a falha terapêutica das infecções causadas por este gênero.

\section{Metodologia}

Esta revisão consistiu na pesquisa de artigos científicos relacionados com o gênero Candida, em bancos de dados de pesquisas científicas consolidados: PubMed/NLM (National Public Library), SciELO (Scientific Electronic Library Online), Lilacs (Literatura Latino-Americana e Caribe em Ciências da Saúde), Web of Science, Science Direct. A pesquisa foi do tipo qualitativa, uma vez que promoveu a prática reflexiva sobre o assunto abordado (Pereira, Shitsuka, Parreira, \& Shitsuka, 2018) e foi realizada entre os meses de janeiro e março de 2020, utilizando os termos: Candida, epidemiologia, candidíase, fatores de virulência, antifúngicos, mecanismos de resistência (Figura 1). 
Figura 1. Estratégia e esquema de pesquisa.

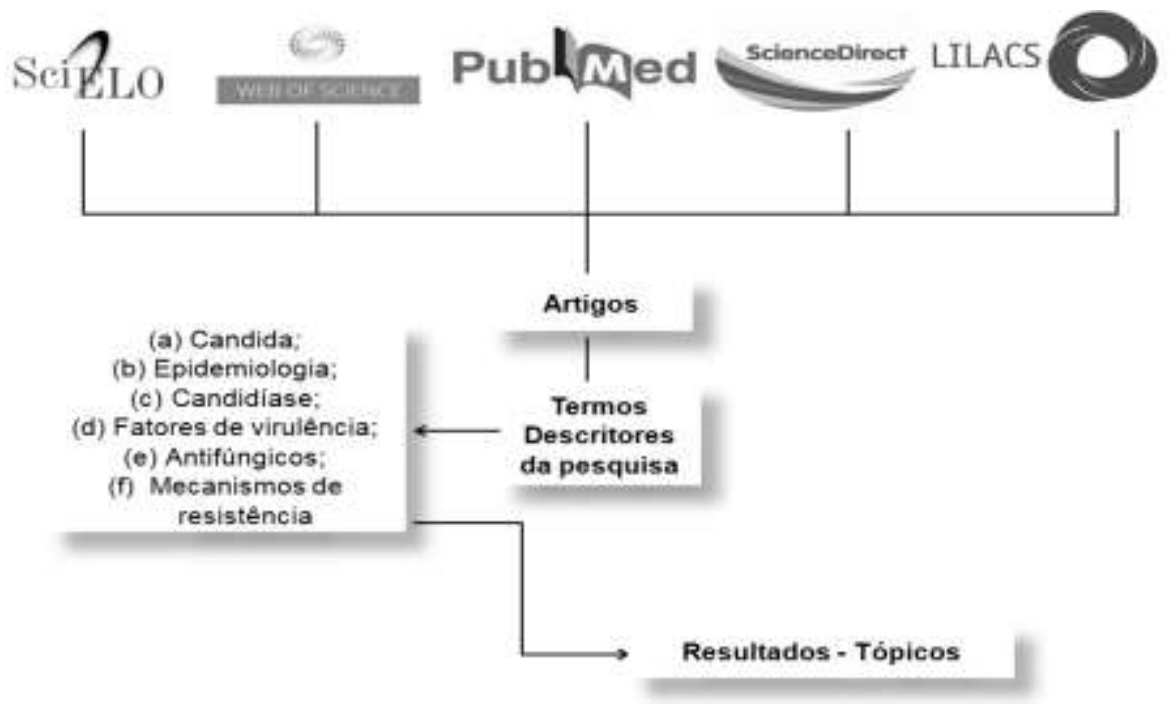

Fonte: Autores (2021).

A seleção de termos relacionados ao nosso objeto de estudo e seus diversos sinônimos foi feita de acordo com MeSH (Medical Subject Headings), DeCS (Descritores em Ciências da Saúde) e palavras de texto e sinônimos dos termos pesquisadas nas bases de dados. Foram incluídos artigos de revisão, artigos de estudos epidemiológicos e artigos de pesquisas laboratoriais entre os anos de 1986 e 2020.

\section{Resultados e Discussão - Revisão de literatura: Fatores de virulência, Epidemiologia, Candidíase e Mecanismos de resistência}

\subsection{Fatores de virulência do gênero Candida}

As espécies do gênero Candida são micro-organismos comensais que se tornam patogênicos, em condições de baixa imunidade do hospedeiro, e, por tais características são considerados patógenos oportunistas (Monod \& Borg-Von Zepelin, 2002).

Para que consigam infectar e colonizar, esses micro-organismos precisam ultrapassar as barreiras de resistência mecânicas (como o tecido epitelial), bioquímicas e físico-químicas (como pH extremos e produção de muco) dos hospedeiros, além da imunidade inata e adquirida (Polke, Hubex \& Jacobsen, 2015; Poulain, 2015).

A espécie C. albicans desenvolveu a capacidade de colonizar diferentes sítios humanos, sendo esse um dos principais motivos para que esta espécie seja a mais incidente em infecções pelo gênero. Esses sítios apresentam diferente microbiota bem como distintas características físico-químicas o que enfatiza sua capacidade de se adaptar a condições inóspitas do sítio de colonização (Calderone \& Clancy, 2011; Polke et al., 2015).

Os principais fatores de virulência relatados para as espécies do gênero Candida incluem: secreção de enzimas hidrolíticas, expressão de adesinas e invasinas, tigmotropismo, bomba de efluxo, formação de biofilme e morfologia celular (Figura 2) (Thompson, Carlisle, \& Kadosh, 2011; Silva et al., 2012; Mayer, Wilson, \& Hube, 2013; Polke et al., 2015). 
Figura 2. Esquema representativo dos principais fatores de virulência do gênero Candida.

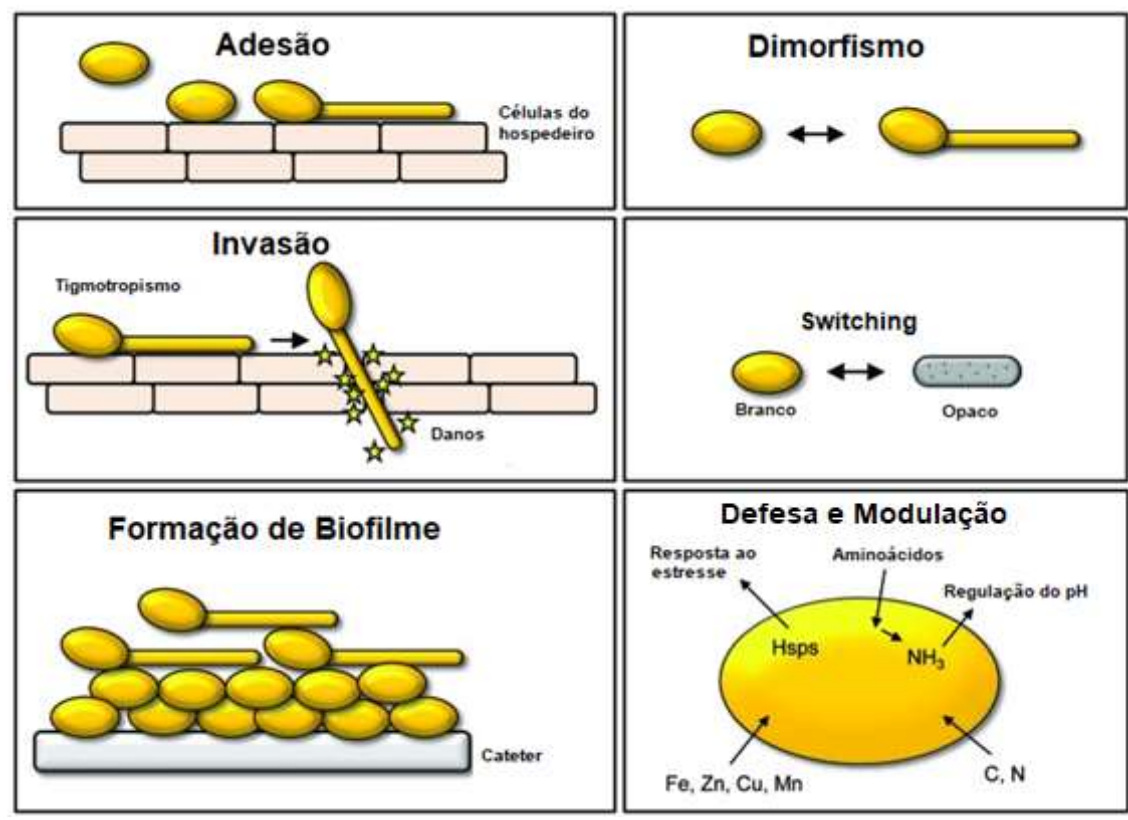

Fonte: Reproduzido/Adaptado de Mayer, Wilson e Hube (2013).

A morfologia celular das espécies deste gênero é citada com um dos principais fatores de virulência, uma vez que as diferentes formas de apresentação celular estão envolvidas em etapas do processo infeccioso. Na etapa de invasão das células do hospedeiro. C. albicans, espécie polimórfica, que se apresenta como células leveduriformes (blastoconídeos) e/ou com crescimento filamentoso na forma de hifas verdadeiras e/ou pseudo-hifas, a morfologia celular, formação de hifas, está diretamente relacionada a capacidade de invasão ao tecido do hospedeiro, enquanto a forma leveduriforme está associada a adesão a célula do hospedeiro (Sudbery, 2011; Thompson et al., 2011; Mayer et al., 2013).

Neste sentido, no processo infeccioso ocorre também a comutação fenotípica que envolve uma variação de células leveduriformes "brancas" para alongadas "opacas", onde as brancas se modificam pelo fenômeno denominado switching, que ocorre quando as células estão em divisão por brotamento ou na formação de biofilmes (Polke et al., 2015).

A secreção de enzimas hidrolíticas, como as fosfolipases, lípases extras e intracelulares estão envolvidas na invasão do epitélio e penetração celular. Dentre as enzimas hidrolíticas biossintetizadas pelo gênero Candida se destacam as proteases aspárticas segregadas (SAPs), que estão envolvidas em processos que destroem, alteram ou danificam a integridade da membrana das células infectadas levando à sua disfunção. São identificadas especificamente em quatro espécies do gênero: $C$. albicans, C. tropicalis, C. parapsilosis e C. dubliniensis. (Monod \& Borg-Von Zepelin, 2002; Schaller, Borelli, Korting \& Hube, 2005; Cauchie, Desmet, \& Lagrou, 2017).

Atualmente, é comum observar que novos estudos estão sendo desenvolvidos a fim de buscar novas moléculas que possam interferer ou inibir a produção/formação destes mecanismos de resistência, principalmente, com enqfoque sobre o biofilme destas espécies (Bezerra, Brito, Cavalcanti, \& Almeida, 2020; Martorano-Fernandes et al., 2021).

\subsection{Epidemiologia das infecções causadas pelo gênero Candida}

O gênero Candida é relatado como a terceira causa das septicemias em geral no mundo (Pappas et al., 2018). Candida albicans continua sendo ao longo dos anos a espécie mais comumente encontrada nas infecções causadas pelas espécies do gênero Candida, prevalecendo em 65,3\% das infecções, por este gênero, ao redor do mundo (Rodrigues, Rodrigues, Silva, \& 
Henriques, 2017; Pfaller et al., 2010). Além da espécie C. albicans, as espécies de Candida não-albicans passaram a ter percentual de incidência relevante, superior a $20 \%$, nos diagnósticos de candidíase (Pfaller et al., 2010).

Pfaller e colaboradores (2010) analisaram os dados do ARTEMIS DISK Global Antifungal Surveillance Study, entre os anos de 1997 e 2007, a ocorrência de espécies do gênero Candida, em diversos sítios de infecção, bem como a sensibilidade aos antifúgicos fluconazol e voriconazol, os dados foram coletados em 41 países distribuídos pelos cinco continentes.

Segundo este estudo, foram identificadas 31 espécies de Candida spp., dentre as espécies mais comumente relatadas de Candida não-albicans foram destaque: C. glabrata (variando de 10,2\% a $11,7 \%$ ao longo dos anos), C. tropicalis (variando de 5,4 \% a 8,0 \%) e C. parapsilosis (variando de 4,8 \% a 5,6 \%). Com relação a resistências das espécies de Candida não-albicans, $15,1 \%$ as cepas de C. glabrata foram resistentes ao fluconazol e 11,3\% resistentes ao voriconazol, com relação as cepas de $C$. krusei, resistência apresentada foi de $66,8 \%$ ao fluconazol e 14,0 \% ao voriconazol (Pfaller et al., 2010).

Os dados da América Latina apontam que a espécie C. albicans correspondeu a 51,8 \% dos isolados, seguida da $C$. tropicalis que atingiu 13,2 \%,C. parapsilosis com 10,3\%,C. glabrata com 7,2\% e C. krusei com 1,4\% de ocorrência. Apesar da $C$. abicans ter sido a mais frequentemente isolada ao longo dos dez anos de estudo, apenas 2,1 e $1,7 \%$ dos isolados na América Latina foi resistente ao fluconazol e voriconazol respectivamente (Pfaller et al., 2010).

Um estudo mais recente demonstrou os resultados do SENTRY Antifungal Surveillance Program, que analisou 1.846 espécies de fungos coletados no ano de 2013, como parte da vigilância global. Os dados evidenciaram, que 1.470 cepas pertenciam ao gênero Candida. Dentre as espécies isoladas deste gênero, 96,2\% estavam distribuídas entre as seguintes espécies: C. albicans (712, 48,4\%), seguida por C. glabrata (251, 17,1\%), C. parapsilosis (215, 14,6\%), C. tropicalis (155, 10,5\%), C. krusei (49, 3,3\%), C. dubliniensis (32,2,2\%), os 3,8\% (56 cepas) estavam distribuídas entre outras espécies do gênero. Quanto ao perfil de resistência dessas cepas se destacaram a $C$. glabrata, onde $2,4 \%$ foram resistentes a anidulafungina e 12,0 \% foram resistentes ao fluconazol, bem como a C. tropicalis que apresentou $11,6 \%$ de resistência ao fluconazol e $1,3 \%$ a anidulafungina, no entanto, as cepas de $C$. albicans não apresentaram resistência a anidulafungina e apresentaram 0,4\% de resistência ao fluconazol (Castanheira et al., 2016).

Existe uma crescente preocupação quanto as espécies de Candida não-albicans, que apesar de serem menos isoladas quando comparadas a $C$. albicans, apresentam importantes perfis de resistência aos antifúngicos disponíveis para tratamento. C. glabrata vem sendo relatada como um dos principais problemas relacionados às infecções causadas por este gênero, uma vez que possui resistência aumentada ao fluconazol, tem alta incidência em adultos, além de apresentar maiores taxas de mortalidade associadas, quando comparada as demais espécies (Rodrigues et al., 2017). Um patógeno emergente, a C. auris tornou-se recentemente um problema, pela expressiva resistência aos agentes antifúngicos como: resistência ao fluconazol em $93 \%$ dos pacientes; a anfotericina B em $35 \%$ e em 7 \% resistentes as equinocandinas (Pappas et al., 2018).

No Brasil, o gênero Candida foi relatado como o sétimo agente etiológico causador das infecções sanguíneas, sendo C. albicans $(34,3 \%)$ a espécie mais prevalente dessas infecções, seguida pela C. parapsilosis (24,1\%), C. tropicalis (15,3\%) e C. glabrata (10,2\%), com uma taxa de mortalidade de 72,2 \% (Doi et al., 2016).

\subsection{Candidíase}

Os micro-organismos do gênero Candida são encontrados convivendo de forma comensal colonizando a microbiota regular das mucosas oral, trato gastrointestinal e geniturinário em cerca de 50-70 \% dos indivíduos saudáveis (Hani et al. 2015). A colonização permite que ambos tenham certas vantagens, dessa forma ao colonizar o ser humano as espécies de Candida conseguem assimilar os nutrientes necessários para sobrevivência. Essa condição limita o crescimento de outros fungos oportunistas, equilibrando a microbiota e promovendo a resposta imunológica (Perlroth, Choi \& Spellberg, 2007; Martins, Ferreira, Barros, Silva, \& Henriques, 2014). 
Em contrapartida, esses micro-organismos podem se tornar patogênicos dependendo de fatores relacionados principalmente ao estado imunológico dos indivíduos, levando a uma infecção oportunista. Dentre os fatores que colaboram para as infecções causadas por Candida podem ser elencados (Perlroth et al., 2007; Vázquez-González, Perusquía-Ortiz, Hundeiker, \& Bonifaz, 2013; Martins et al., 2014; Hani et al. 2015; Pappas et al. 2018):

a. Indivíduos imunossuprimidos (pacientes acometidos por síndrome da imunodeficiência adquirida - SIDA, pacientes em uso de imunossupressores; câncer e diabetes);

b. Utilização de antibióticos de amplo espectro para tratamento e utilização preventiva, desequilibrando a microbiota;

c. Procedimentos cirúrgicos invasivos, uso prolongado de cateteres e hospitalização prolongada;

d. Cirurgias de transplantes;

e. Velhice, gravidez e crianças de nascimento prematuro;

f. Reposição hormonal e uso de hormônios esteróides.

De acordo com Knoke e Bernhardt (2006) a primeira vez que o gênero Candida foi reconhecido como patógeno humano foi em 1839 por Bernhard von Langenbeck, quando a espécie C. albicans foi isolada de uma infecção oral de um paciente acometido por tifo.

Comumente nestas condições, esses micro-organismos se tornam patógenos humanos e causam infecções que são denominadas de Candidíase. Candidíase é um termo usado amplamente para denominar infecções causadas por fungos do gênero Candida sejam elas mucocutâneas ou superficiais, bem como infecções invasivas de órgãos profundos ou sangue (candidemia) (Ruping, Vehreschild, \& Cornely, 2008; Silva et al., 2012; Hani et al. 2015; Pappas et al. 2018). Porém, segundo Martins e colaboradores (2014), nas últimas décadas vêm sendo identificados casos de candidíase em indivíduos saudáveis e que não estão enquadrados nestes fatores predisponentes.

A candidíase mucocutânea ou superficial acomete pele, cabelos, unhas e membranas mucosas. A candidíase oral, que pode ser classificada como intraoral, faríngea e perioral, pelo estágio agudo ou crônico e acomete preferencialmente os indivíduos com SIDA, idosos com próteses dentárias e fumantes (Vázquez-González et al., 2013; Hellstein \& Marek, 2019).

A candidíase vulvovaginal acomete cerca de $50 \%$ das mulheres (com casos de recorrência), e pode atingir bebês do sexo feminino neonatas; a candidíase postular afeta usuários de drogas, mas que também pode ocorrer em pacientes hospitalizados e/ou imunossuprimidos, acometendo a pele e o couro cabeludo (Vázquez-González et al., 2013). A onicomicose que atinge as unhas e pode ser provocado também por outros gêneros de fungos, acomete em geral pacientes com SIDA, diabético ou provocado por traumas ocorridos em manicures (Silva, Lima, Santos, \& Lima, 2020; Cambuim et al., 2011; Vázquez-González et al., 2013).

As infecções invasivas causadas por espécies do gênero Candida podem atingir o sangue, sendo a infecção denominada de Candidemia, ou atingir outros órgãos e/ou tecidos profundos, como o estômago (peritonite, abscesso intraabdominal), ossos (osteomielite), olhos, cérebro, coração, rim, fígado e pulmões (pneumonia). Essas infecções de órgãos profundos estão mais frequentemente associadas a candidemia, porém a exemplo da peritonite podem aparecer associados ou não a esta condição (Kullberg \& Arendrup, 2015; Dermawan, Ghosh, Keating, Gopalakrishna, \& Mukhopadhyay, 2018; Pappas et al., 2018).

As cepas colonizadoras podem invadir o tecido após adesão inicial de forma endógena, dependendo das condições do paciente e das características da espécie, e se disseminar pela corrente sanguínea. Na sua forma exógena, pode ser veiculada pela utilização de cateteres venosos desencadeando a formação de biofilme nestes instrumentos, levando também a candidemia (Kullberg \& Arendrup, 2015; Pappas et al., 2018). 


\subsection{Antifúngicos - Classificação e Mecanismos de ação}

\subsubsection{Antifúngicos Azóis}

Os antifúngicos azóis agem inibindo a enzima do citocromo P450 14 $\alpha$-lanosterol desmetilase que participa de uma das etapas mais importantes da rota da biossíntese de ergosterol nas leveduras e fungos filamentosos. Nas espécies do gênero Candida essa enzima é codificada pelo gene ERG11, sendo uma das dez enzimas responsáveis pela etapa última etapa da rota de conversão de lanosterol em ergosterol (Akins, 2005; (Prasad, Shah, \& Rawal, 2016).

A enzima $14 \alpha$-lanosterol desmetilase possui uma porção heme em seu sítio ativo, o nitrogênio da molécula dos azóis reage com o átomo de ferro contido na porção heme da enzima, inibindo a ativação do oxigênio que é necessário na reação de desmetilação do lanosterol em ergosterol pela atividade da enzima. Em decorrência do bloqueio na biossíntese de ergosterol ocorre acúmulo de um esterol tóxico para a membrana celular, denominado de 14- $\alpha$-metil-3,6-diol, que causa estresse celular e não permite a continuidade do ciclo celular do micro-organismo (Shapiro, Robbins, \& Cowen, 2011; Hani et al. 2015). Os azólicos atuam também pela inibição na transformação das células leveduriformes de Candida em hifas e/ou pseudo-hifas, que constituem um dos principais fatores de virulência, sendo a forma invasiva e patogênica mais expressiva do gênero (Rang, Dale, Ritter, \& Moore, 2007).

\subsubsection{Antifúngicos Alilaminas}

Esses antifúngicos agem inibindo a biossíntese de ergosterol através da inibição da enzima escaleno epoxidase, codificada pelo gene ERG1, que é responsável por converter escaleno em lanosterol, impedindo a biossíntese de ergosterol da membrana. A inibição da esqualeno epoxidase resulta em o acúmulo de esqualeno dentro da célula e um declínio no conteúdo de ergosterol, sendo esses eventos os responsáveis pelo bloqueio do crescimento fungico (Akins, 2005; Rang et al., 2007). Para as espécies de Candida em especial a C. albicans é relatado que a forma de hifas/pseudohifas é cerca de dez vezes mais suscetível a inibição por alilaminas, do que a forma de levedura (Birnbaum, 1990).

\subsubsection{Antifúngicos Polienos}

O principal mecanismo de ação dos polienos esta definido como a interação com o componente majoritário da membrana celular dos fungos, o ergosterol. Essa interação promove a formação de poros transmembrana, criando um canal iônico que consequentemente causa vazamento e perda de íons intracelulares (Prasad et al., 2016; (Kristanc, Božič, Jokhadar, Dolenc, \& Gomišček, 2019).

Os poros transmembranares são formados em decorrência da estrutura anfipática característica dos polienos: a cadeia poliênica que representa a parte hidrofóbica da molécula interage com as moléculas de esterol da membrana, enquanto que a parte hidrofílica, representada pela cadeia poliol fica no ambiente aquoso, formando um poro transmembranar, que causa extravasamento do conteúdo intracelular, em particular íons monovalentes como $\mathrm{K}^{+}, \mathrm{Na}^{+}$e $\mathrm{Cl}^{-}$, resultando em um desequilíbrio eletroquímico (Cohen, 2010; Serhan, Stack, Perrone, \& Morton, 2014).

\subsubsection{Equinocandinas - Inibidores da parede celular fúngica}

As equinocandinas agem por um único mecanismo de ação, inibindo a síntese da parede celular de fungos, através da inibição não competitiva da enzima (1,3)- $\beta$-D-glucana sintase, essa enzima é responsável pela síntese do polímero $(1,3)-\beta$-Dglucana, constituinte importante da parede celular, que se ligam covalentemente ao outro polímero a (1,6)- $\beta$-D-glucana, e juntos com a quitina formam uma rede tridimensional complexa de microfibrilas. A inibição da enzima promove inibição de biossíntese da parede celular, causando lise e prejuízo a integridade celular (Onishi et al., 2000; Perlin, 2011; Emri, Majoros, Tóth, \& Pócsi, 2014); Prasad et al.,2016). 


\subsubsection{Análogos da pirimidina - ação no material genético}

A 5-fluorocitocina (5-FC) ou flucitocina é o representante desta classe conhecida por sua atividade antifúngica e antineoplásica, embora novos análogos da 5-FC estejam sendo obtidos (Wani, Ahmad, Kumar, \& Sobral, 2017). Segundo Vermes e colaboradores (2000), o 5-FU age nas células fúngicas por duas vias: 1. Pela conversão de 5-FU em trifosfato de 5fluorouridina que ao ser incorporado ao RNA do fungo causa modificações na sequência de aminoácidos e inibição da síntese de proteínas e/ou 2. Pela conversão de 5-FU em monofosfato de 5-fluorodeoxiuridina que inibe a enzima timidilato sintetase, impedindo a biossíntese de DNA. Dessa forma essa droga só funciona em espécies que possuem a enzima citosina desaminase, gerando o metabólito 5-FU responsável pela ação antifúngica, tanto que não apresenta tal ação nas células humanas, já que a enzima citosina desaminase não está presente (Rang et al., 2007).

A Figura 3 apresenta o esquema de mecanismos de ação das principais classes de antifúngicos.

Figura 3. Mecanismos de ação das principais classes de antifúngicos.

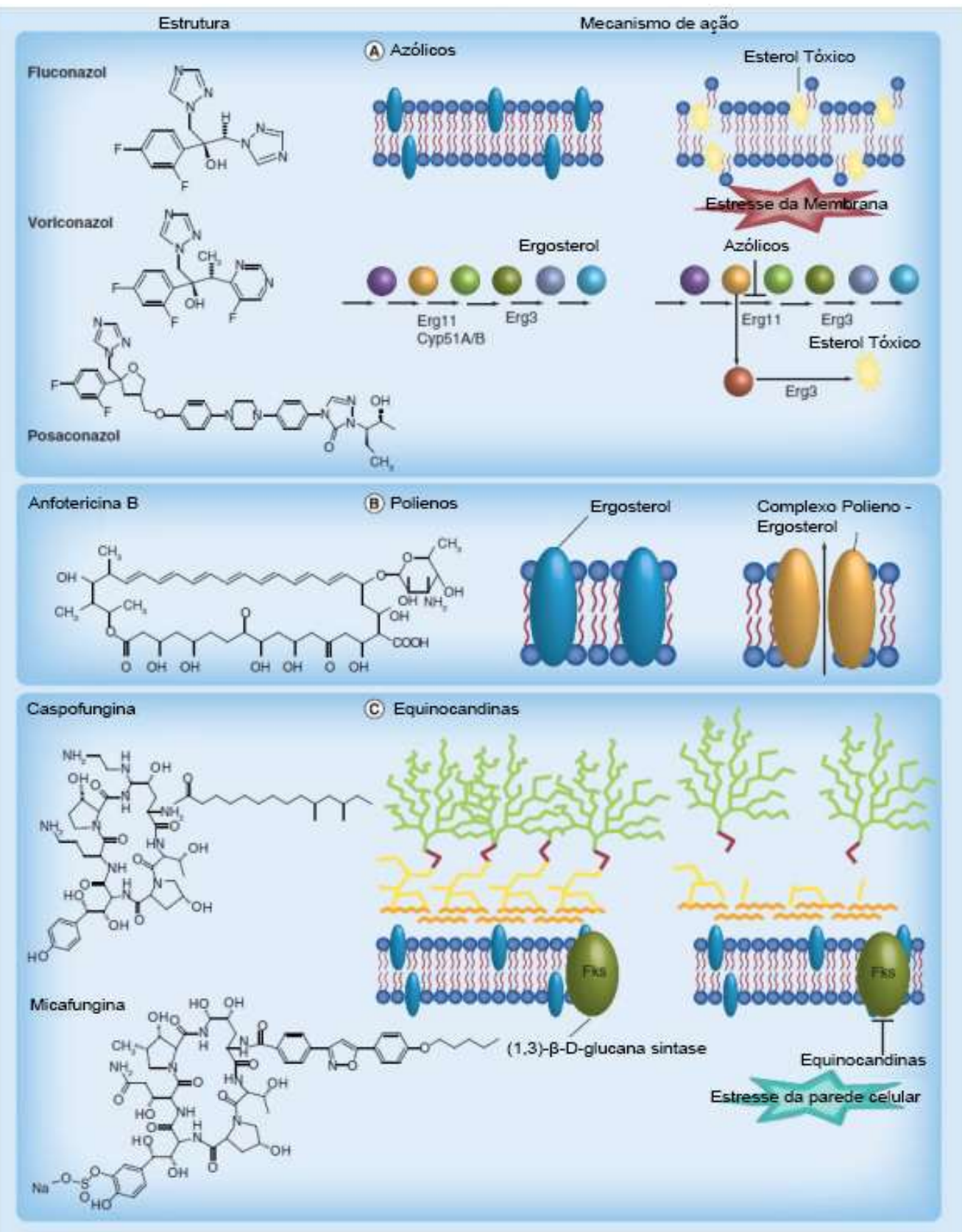

Fonte: Reproduzido/Adaptado de Xie, Polvi, Shekhar-Guturja, e Cowen (2014). 


\subsection{Mecanismos de resistência do gênero Candida aos antifúngicos}

\subsubsection{Resistência aos azóis}

Os mecanismos atribuídos a resistência aos azóis tanto em C. albicans como espécies Candida não-albicans estão bem definidos e estão associados principalmente a expressão aumentada da bomba de efluxo de drogas, a maior expressão e modificação na estrutura de sítios alvos, além da formação de biofilme (Figura 4). Esses mecanismos podem ocorrer isolados ou em conjunto, sendo que a expressão aumentada da bomba de efluxo de drogas é relatada como a causa primária da resistência aos azóis em espécies de Candida. A expressão da bomba de efluxo resulta na baixa concentração do agente antifúngico no interior da célula fúngica, conferindo resistência a essas drogas (Sanglard et al., 1995; Bulatova \& Darwish, 2008; Perlin, Rautemaa-Richardson, \& Alastruey-Izquierdo, 2017).

Para as espécies de Candida são relatadas duas grandes famílias de domínios proteicos transmembrana que funcionam como bombas de efluxo de drogas. Uma delas é a superfamília MFS - Major facilitador superfamily, porém a mais estudada é a superfamília dos transportadores ABC - ATP-binding cassete transporters, ambas utilizam diferentes fontes de energia para seu funcionamento efetivo no efluxo de drogas do meio intracelular para o meio extracelular de espécies de Candida e sua expressão garante resistência a determinadas drogas (Cannon et al., 2009).

Outro importante mecanismo de resistência a esta classe de drogas ocorre pela expressão aumentada do gene ERG11 que participa na regulação da síntese de ergosterol. O gene ERG11 codifica a enzima alvo dos antifúngicos azóis, a lanosterol $14 \alpha$-desmetilase, ao entrar em contato com o antifúngico a depleção do ergosterol faz com que o micro-organismo regule de forma positiva o gene ERG11 aumentando a presença de sítios alvo (Henry, Nickels, \& Edlind, 2000; Kanafani \& Perfect, 2008). Henry e colaboradores (2000) relataram que esse fenômeno ocorreu após a exposição de C. albicans, C. glabrata, C. krusei e C. tropicalis ao tratamento com fluconazol, itraconazol, cetoconazol, miconazol e clotrimazol, ocorrendo uma regulação positiva do gene ERG11.

A modificação na estrutura da enzima alvo lanosterol $14 \alpha$-desmetilase também é citada como causa da resistência aos azóis. As mutações garantiram modificações no sítio alvo a ponto de modificar a afinidade do antifúngico azólico a enzima e dessa forma não desempenha mais sua atividade diante de cepas de C. albicans (Sanglard, Ischer, Koymans, \& Bille, 1998; Marichal et al.,1999).

A formação de biofilme pelas espécies de Candida spp. também é citado como um importante mecanismo para a falta de ação dos antifúngicos azolicos, sendo um mecanismo de resistência comum também as demais classes de antifúngicos, eles representam um importante desafio para o tratamento de infecções crônicas sendo necessário a busca por novos alvos terapêuticos e estratégias de combinação de drogas na busca de um mais tratamento efetivo (Koo, Allan, Howlin, Stoodley, \& Hall-Stoodley; 2017).

Biofilmes maduros apresentam uma estrutura bem consolidada, com substâncias denominadas de exopolissacarídeos (EPS - extracellular polymeric substance) constituindo uma matriz extracelular forte que promove a ligação das células a uma superfície, ligação entre células e agregação arquitetada. Para as espécies de Candida essa matriz é constituída em geral de carboidratos, proteínas, hexosamina, fósforo e ácido urônico. Sejam esses biofilmes de um mesmo micro-organismo ou de dois ou mais desses, essa matriz é responsável por manter uma barreira mecânica estável e também proteger os micro-organismos de as drogas antimicrobianas e, portanto, desempenham papel na resistência a essas drogas (Al-Fattani \& Douglas, 2006; Flemming \& Wingender, 2010; Koo et al., 2017; Perlin et al., 2017). 
Figura 4. Representação esquemática dos principais mecanismos de resistência do gênero Candida aos antifúngicos.

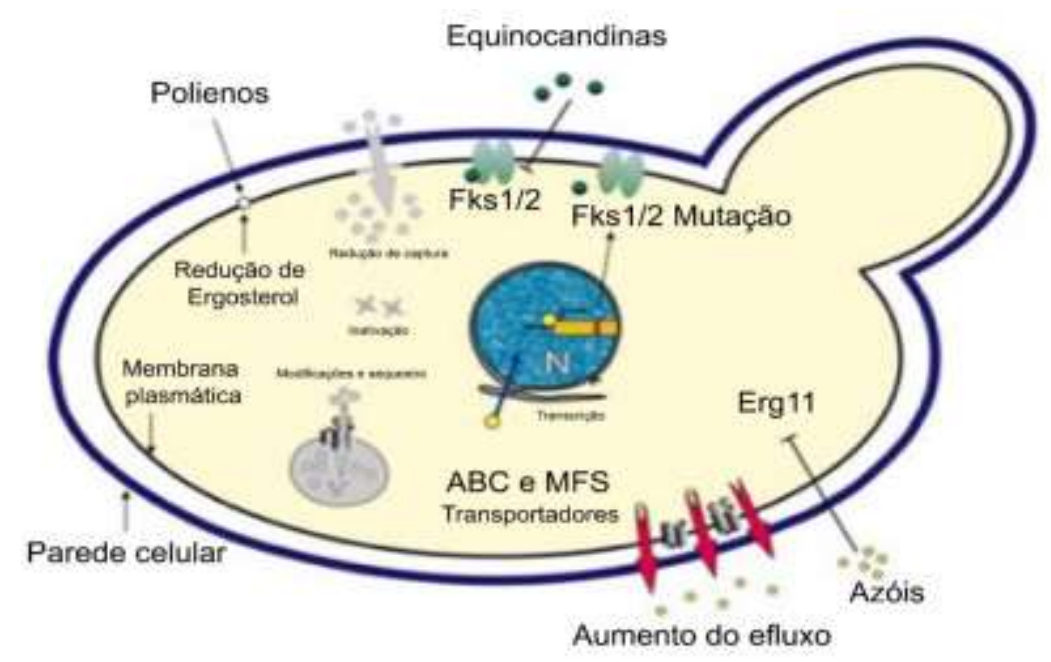

Fonte: Reproduzido/Adaptado de Tscherner, Schwarzmüller e Kuchler (2011).

\subsubsection{Resistência as equinocandinas}

A resistência aos antifúngicos da classe das Equinocandinas ainda é bastante incomum entre as espécies de Candida. Essa resistência incomum parece estar envolvida com a mutação do gene FKS (Figura 4), através da substituição de aminoácidos em duas regiões hot spot de Fks1 para todas as espécies de Candida e Fks2 em C. glabrata (Park et al., 2005; Balashov, Park, \& Perlin, 2006; Perlin, 2011; Perlin et al., 2017; Kordalewska et al., 2018; Pristov \& Ghannoum, 2019). Essas mutações se manifestam pelo aumento das concentrações inibitórias mínimas das cepas de Candida e consequentemente na falha no tratamento.

O gene FKS codifica a enzima 1,3- $\beta$-glucana sintetase, que é o alvo de ação das echinocandinas e é responsável pela

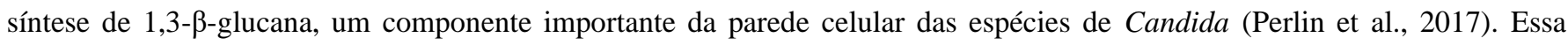
mutação do gene $F K S$ em espécies de Candida pode estar relacionada ao estresse provocado pela exposição prolongada, exposição repetidas vezes a drogas desta classe, ou o conjunto desses dois fatores. Porém estão também relacionadas ao polimorfismo natural de certas espécies de Candida, cmo é o caso da C. parapsilosis e da C. guilliermondii, caracterizada pelos valores de concentração inibitória mínina bem mais elevados quando comparado a cepas susceptíveis a esta classe (Pfaller et al., 2010; Perlin et al., 2017).

Segundo Niimi e colaboradores (2006), a expressão de genes codificadores de bomba de efluxo (CDR1, CDR2, MDR1 e MDR2), que garantem resistência aos azóis, não parecem interferir na susceptibilidade das cepas de Candida as equinocandinas.

\subsubsection{Resistência aos polienos}

Os polienos agem pela interação com o ergosterol presente na membrana plasmática das células de Candida spp. formando poros que ligam o interior da célula com o meio externo e fazem o micro-organismo perder o seu equilíbrio osmótico e os nutrientes essenciais a sua sobrevivência. Mutações nos genes ERG2, ERG3, ERG6 e ERG11 (Figura 4) parecem estar associados a resistência das espécies de Candida aos polienos, uma vez que ocorre uma diminuição da expressão do alvo de ação dessas drogas (Vandeputte et al., 2007; Vincent, Lancaster, Scherz-Shouval, Whitesell, \& Lindquist, 2013; Eddouzi et al., 2013; Prasad et al., 2016).

Dessa forma, os isolados de Candida que apresentam um teor mais baixo de ergosterol tendem a ser resistentes ao polienos, quando comparado com o teor de ergosterol de cepas susceptíveis a estas drogas (Dick, Merz \& Saral, 1980), essa 
característica pode estar associada a mutações dos genes reguladores da síntese de ergosterol, bem como ao tratamento prévio com antifúngicos inibidores da síntese de ergosterol, como é o caso dos agentes azóis (Vazquez et al. 1998; Xie et al., 2014).

Existe uma correlação da resistência aos polienos com a resistência aos agentes azóis, quando estes se relacionam com as modificações nos genes responsáveis pela síntese de ergosterol (em destaque o ERG3), decréscimo de ergosterol na membrana e acúmulo de ergosterol alternativo (Kelly, Lamb, Kelly, Loeffler \& Einsele, 1996; Kelly et al., 1997; Eddouzi et al., 2013). Existe ainda uma correlação da resistência a anfotericina B em biofilmes pré-formados, bem como a falta da capacidade dessa droga de não impeder a produção de biofilme de $C$. tropicalis (Fernandes, Silva, \& Henriques, 2015). SokolAnderson, Brajtburg, e Medoff (1986) relacionaram também o aumento da atividade da enzima catalase endógena mediando a resistência à anfotericina diminuindo a susceptibilidade de cepas de C. albicans.

\section{Considerações Finais}

As espécies do gênero Candida representam um problema de saúde, pelo surgimento de cepas e de novas espécies cada vez mais resistentes aos antifúngicos disponíveis para o tratamento de infecções causadas por estes micro-organismos, o que torna estas infecções muitas vezes não tratáveis, trazendo maléficos para o paciente.

Dessa forma é necessária atualização constante dos tratamentos disponíveis, os principais mecanismos de resistência deste gênero aos antifúngicos bem como os aspectos relacionados a candidíase, seja invasiva ou superficial, sendo possível compreender e direcionar as pesquisas para busca de novos antifúngicos, com enfoque em novos alvos terapêuticos, estratégias de prevenção e tratamento destas infecções.

\section{Referências}

Akins, R. A. (2005). An update on antifungal targets and mechanisms of resistance in Candida albicans. Medical Mycology, 43(4), 285-318. https://doi.org/10.1080/13693780500138971

Al-Fattani, M. A., \& Douglas, L. J. (2006). Biofilm matrix of Candida albicans and Candida tropicalis: chemical composition and role in drug resistance. Journal of Medical Microbiology, 55(8), 999-1008. https://doi.org/10.1099/jmm.0.46569-0

Balashov, S. V., Park, S., \& Perlin, D. S. (2006). Assessing resistance to the echinocandin antifungal drug caspofungin in Candida albicans by profiling mutations in FKS1. Antimicrobial Agents of Chemotherapy, 50, 2058-2063. https://doi.org/10.1128/AAC.01653-05

Birnbaum, J. E. (1990). Pharmacology of the allylamines. Journal of the American Academy of Dermatology, 23(4), 782-785. https://doi.org/10.1016/01909622(90)70288-s

Bezerra, I. M., Brito, A. C. M., Cavalcanti, Y. W., Almeida, L. F. D. (2020). Inhibitory effect of phytochemicals on Candida albicans biofilms on denture liners surfaces. Research, Society and Development, 9(10), p. e2279108587. https://doi.org/10.33448/rsd-v9i10.8587.

Bulatova, N. R., \& Darwish, R. M. (2008). Effect of chemosensitizers on minimum inhibitory concentrations of fluconazole in Candida albicans. Medical Principles and Practice, 17(2), 117-121. https://doi.org/10.1159/000112964

Calderone, R. A., \& Clancy, C. J. (Eds.). (2011). Candida and candidiasis. American Society for Microbiology Press. Disponível em: https://books.google.com.br/

Cambuim, I. I., Macêdo, D. P., Delgado, M., Lima, K. M., Mendes, G. P., Souza-Motta, C. M., Lima, D. M., Fernandes, M. J., Magalhães, O. M., Queiroz, L. A., \& Neves, R. P. (2011). Clinical and mycological evaluation of onychomycosis among Brazilian HIV/AIDS patients. Revista da Sociedade Brasileira de Medicina Tropical, 44(1), 40-42. https://doi.org/10.1590/s0037-86822011000100010

Castanheira, M., Messer, S. A., Rhomberg, P. R., \& Pfaller, M. A. (2016). Antifungal susceptibility patterns of a global collection of fungal isolates: results of the SENTRY Antifungal Surveillance Program (2013). Diagnostic Microbiology and Infectious Disease, 85, 200-204. https://doi.org/10.1016/j.diagmicrobio.2016.02.009

Cannon, R. D., Lamping, E., Holmes, A. R., Niimi, K., Baret, P. V., Keniya, M. V., Tanabe K., Niimi, M., Goffeau, A., \& Monk, B. C. (2009). Effluxmediated antifungal drug resistance. Clinical microbiology reviews, 22(2), 291-321. https://doi.org/10.1128/CMR.00051-08

Cauchie, M., Desmet, S., \& Lagrou, K. (2017). Candida and its dual lifestyle as a commensal and a pathogen. Research in Microbiology, 168(9-10), 802-810. https://doi.org/10.1016/j.resmic.2017.02.005

Cohen, B. E. (2010). Amphotericin B membrane action: role for two types of ion channels in eliciting cell survival and lethal effects. The Journal of Membrane Biology, 238(1-3), 1-20. https://doi.org/10.1007/s00232-010-9313-y 
Dermawan, J. K. T., Ghosh, S., Keating, M. K., Gopalakrishna, K. V., \& Mukhopadhyay, S. (2018). Candida pneumonia with severe clinical course, recovery with antifungal therapy and unusual pathologic findings: A case report. Medicine, 97(2), e9650. https://doi.org/10.1097/MD.0000000000009650

Dick, J. D., Merz, W. G., \& Saral, R. (1980). Incidence of polyene-resistant yeasts recovered from clinical specimens. Antimicrobial Agents of Chemotherapy, 18, 158-63. https://doi.org/10.1128/aac.18.1.158

Doi, A. M., Pignatari, A. C., Edmond, M. B., Marra, A. R., Camargo, L. F., Siqueira, R. A., Mota, V. P., \& Colombo, A. L. (2016). Epidemiology and microbiologic characterization of nosocomial candidemia from a Brazilian national surveillance program. PloS one, 11(1), e0146909. https://doi.org/10.1371/journal.pone.0146909

Eddouzi, J., Parker, J. E., Vale-Silva, L. A., Coste, U. M., Ischer, F., Kelly, S., Manai, H., \& Sanglard, D. (2013). Molecular mechanisms of drug resistance in clinical Candida species isolated from Tunisian hospitals. Antimicrobial Agents of Chemotherapy, 57(7), 3182-3193. https://doi.org/10.1128/AAC.00555-13

Emri, T., Majoros, L., Tóth, V., \& Pócsi, I. (2013). Echinocandins: production and applications. Applied Microbiology and Biotechnology, 97(8), 3267-3284. https://doi.org/10.1007/s00253-013-4761-9

Fernandes, T., Silva, S., \& Henriques, M. (2015). Candida tropicalis biofilm's matrix-involvement on its resistance to amphotericin B. Diagnostic Microbiology and Infectious Disease, 83(2), 165-169. https://doi.org/10.1016/j.diagmicrobio.2015.06.015

Flemming, H. C., \& Wingender, J. (2010). The biofilm matrix. Nature Reviews Microbiology. 8, 623-633. https://doi.org/10.1038/nrmicro2415

Hani, L., Shivakumar, H. G., Vaghela, R., Osmani, R. A., \& Shrivastava, A. (2015). Candidiasis: a fungal infection-current challenges and progress in prevention and treatment. Infectious Disorders-Drug Targets, 15, 42-52. https://doi.org/10.2174/1871526515666150320162036

Hellstein, J. W., \& Marek, C. L. (2019). Candidiasis: Red and White Manifestations in the Oral Cavity. Head and Neck Pathology, 13(1), 25-32. https://doi.org/10.1007/s12105-019-01004-6

Henry, K. W., Nickels, J. T., \& Edlind, T. D. (2000). Upregulation of ERG genes in Candida species by azoles and other sterol biosynthesis inhibitors. Antimicrobial Agents and Chemotherapy, 44(10), 2693-2700. https://doi.org/10.1128/aac.44.10.2693-2700.2000

Kanafani, Z. A., \& Perfect, J. R. (2008). Resistance to antifungal agents: mechanisms and clinical impact. Clinical infectious diseases, 46(1), 120-128. https://doi.org/10.1086/524071

Kelly, S. L.; Lamb, D. C.; Kelly, D. E.; Loeffler, J.; Einsele, H. (1996). Resistance to fluconazole and amphotericin in Candida albicans from AIDS patients. Lancet, 348(9040), 1523-1524. https://doi.org/10.1016/S0140-6736(05)65949-1

Kelly, S. L.; Lamb, D. C.; Kelly, D. E.; Manning, N. J.; Loeffler, J.; Hebart, H.; Schumacher, U.; Einsele H. (1997). Resistance to fluconazole and crossresistance to amphotericin B in Candida albicans from AIDS patients caused by defective sterol delta-5,6-desaturation. FEBS Letters, 400(1), 80-82. https://doi.org/10.1016/s0014-5793(96)01360-9

Kristanc, L., Božič, B., Jokhadar, Š. Z., Dolenc, M. S., x Gomišček, G. (2019). The pore-forming action of polyenes: From model membranes to living organisms. Biochimica et Biophysica Acta (BBA)-Biomembranes, 1861(2), 418-430. https://doi.org/10.1016/j.bbamem.2018.11.006

Koo, H., Allan, R. N., Howlin, R. P., Stoodley, P., \& Hall-Stoodley, L. (2017). Targeting microbial biofilms: current and prospective therapeutic strategies. Nature Reviews Microbiology, 15(12), 740. https://doi.org/10.1038/nrmicro.2017.99

Kordalewska, M., Lee, A., Park, S., Berrio, I., Chowdhary, A., Zhao, Y., \& Perlin, D. S. (2018). Understanding echinocandin resistance in the emerging pathogen Candida auris. Antimicrobial Agents and Chemotherapy, 62(6), e00238-18. https://doi.org/10.1128/AAC.00238-18

Knoke, M., \& Bernhardt, H. (2006). The first description of an oesophageal candidosis by Bernhard von Langenbeck in 1839. Mycoses, 49(4), 283-287. https://doi.org/10.1111/j.1439-0507.2006.01237.x

Kullberg, B. J., \& Arendrup, M. C. (2015). Invasive candidiasis. New England Journal of Medicine, 373(15), 1445-1456. https://doi.org/10.1056/NEJMra1315399

Mayer, F. L., Wilson, D., \& Hube, B. (2013). Candida albicans pathogenicity mechanisms. Virulence, 4(2), 119-128. https://doi.org/10.4161/viru.22913

Martins, N., Ferreira, I. C., Barros, L., Silva, S., \& Henriques, M. (2014). Candidiasis: predisposing factors, prevention, diagnosis and alternative treatment. Mycopathology, 177(5-6), 223-240. https://doi.org/10.1007/s11046-014-9749-1

Martorano-Fernandes, L., Rodrigues, N. C., Bezerra, N. V. F., Borges, M. H. S., Cavalcanti, Y. W., Almeida, L. F. D. (2021). Cinnamaldehyde and $\alpha$-terpineol as an alternative for using as denture cleansers: antifungal activity and acrylic resin color stability. Research, Society and Development, 10(3), e28010313512, https://doi.org/10.33448/rsd-v10i3.13512.

Marichal, P., Koymans, L., Willemsens, S., Bellens, D., Verhasselt, P., Luyten, W., Borgers, M., Ramaekers, F. C. S., Odds, F. C., Vanden Bossche, H. (1999). Contribution of mutations in the cytochrome P450 14 $\alpha$-demethylase (Erg11p, Cyp51p) to azole resistance in Candida albicans. Microbiology, 145(10), 2701-2713. https://doi.org/10.1099/00221287-145-10-2701

Monod, M., \& Borg-Von Zepelin, M. (2002). Secreted aspartic proteases as virulence factors of Candida species. Biological Chemistry, 383(7-8), 1087-1093. https://doi.org/10.1515/BC.2002.117

Modrzewska, B., \& Kurnatowski, P. (2013). Selected pathogenic characteristics of fungi from the genus Candida. Annals Parasitology, 59(2), 57-66. PMID: 24171298

Niimi, K., Maki, K., Ikeda, F., Holmes, A. R., Lamping, E., Niimi, M., Monk, B. C., \& Cannon, R. D. (2006). Overexpression of Candida albicans CDR1, CDR2, or MDR1 does not produce significant changes in echinocandin susceptibility. Antimicrobial Agents and Chemotherapy, 50, 1148-1155. https://doi.org/10.1128/AAC.50.4.1148-1155.2006 
Onishi, J., Meinz, M., Thompson, J., Curotto, J., Dreikorn, S., Rosenbach, M., Douglas, C., Abruzzo, G., Flattery, A., Kong, L., Cabello, A., Vicente, F., Pelaez, F., Diez, M. T., Martin, I., Bills, G., Giacobbe, R., Dombrowski, A., Schwartz, R., Morris, S., Harris, G., Tsipouras, A., Wilson, K., \& Kurtz, M. B. (2000). Discovery of novel antifungal (1, 3)- $\beta$-D-glucan synthase inhibitors. Antimicrobial Agents and Chemotherapy, 44(2), 368-377. https://doi.org/10.1128/aac.44.2.368-377.2000

Pappas, P. G., Lionakis, M. S., Arendrup, M. C., Ostrosky-Zeichner, L., \& Kullberg, B. J. (2018). Invasive candidiasis. Nature Reviews Disease Primers, 4 , 18026. https://doi.org/10.1038/nrdp.2018.26

Park, S., Kelly, R., Kahn, J. N., Robles, J., Hsu, M. J., Register, E., Li, W., Vyas, V., Fan, H., Abruzzo, G., Flattery, A., Gill, C., Chrebet, G., Parent, S. A., Kurtz, M., Teppler, H., Douglas, C. M., \& Perlin, D. S. (2005). Specific substitutions in the echinocandin target Fks1p account for reduced susceptibility of rare laboratory and clinical Candida sp. isolates. Antimicrobial Agents and Chemotherapy, 49, 3264-3273. https://doi.org/10.1128/AAC.49.8.3264-3273.2005

Pereira, A. S., Shitsuka, D. M., Parreira, F. J., \& Shitsuka, R. (2018). Metodologia da pesquisa científica. UFSM. https://repositorio. ufsm. br/bitstream/handle/1/15824/Lic_Computacao_Metodologia-Pesquisa-Cientifica. pdf.

Perlin, D. S. (2011). Current perspectives on echinocandin class drugs. Future Microbiology, 6, 441-457. https://doi.org/10.2217/fmb.11.19

Perlin, D. S., Rautemaa-Richardson, R., \& Alastruey-Izquierdo, A. (2017). The global problem of antifungal resistance: prevalence, mechanisms, and management. The Lancet Infectious Diseases, 17, e383-e392. https://doi.org/10.1016/S1473-3099(17)30316-X

Perlroth, J., Choi, B., \& Spellberg, B. (2007). Nosocomial fungal infections: epidemiology, diagnosis, and treatment. Medical Mycology, 45, 321-346. https://doi.org/10.1080/13693780701218689

Pfaller, M. A., Diekema, D. J., Gibbs, D. L., Newell, V. A., Ellis, D., Tullio, V., Rodloff, A., Fu, W., Ling, T. A., \& the Global Antifungal Surveillance Group. (2010). Results from the ARTEMIS DISK Global Antifungal Surveillance Study, 1997 to 2007: a 10.5-year analysis of susceptibilities of Candida species to fluconazole and voriconazole as determined by CLSI standardized disk diffusion. Journal of Clinical Microbiology, 48, 1366-1377. https://doi.org/10.1128/JCM.02117-09

Polke, M., Hube, B., \& Jacobsen, I. D. (2015). Candida survival strategies. Advances in Applied Microbiology, 91, 139-235. https://doi.org/10.1016/bs.aambs.2014.12.002

Poulain, D. (2015). Candida albicans, plasticity and pathogenesis. Critical Reviews in Microbiology, 41(2), 208-217. https://doi.org/10.3109/1040841X.2013.813904

Prasad, R., Shah, A. H., \& Rawal, M. K. (2016). Antifungals: mechanism of action and drug resistance. Yeast Membrane Transport, p. 327-349. https://doi.org/10.1007/978-3-319-25304-6_14

Pristov, K. E., \& Ghannoum, M. A. (2019). Resistance of Candida to azoles and echinocandins worldwide. Clinical Microbiology and Infection, 25(7),792798. https://doi.org/10.1016/j.cmi.2019.03.028

Rang, H. P., Dale, M. M., Ritter, J. M., \& Moore, P. K. (2007). Farmacologia. (6a ed.), Guabanara, Koogan AS.

Rodrigues, C. F., Rodrigues, M. E., Silva, S., \& Henriques, M. (2017). Candida glabrata biofilms: how far have we come? Journal of Fungi, 3 , e11. https://doi.org/10.3390/jof3010011

Ruping, M. J., Vehreschild, J. J., \& Cornely, O. A. (2008). Patients at high risk of invasive fungal infections: when and how to treat. Drugs, 68, $1941-1962$. https://doi.org/10.2165/00003495-200868140-00002

Sanglard, D., Kuchler, K., Ischer, F., Pagani, J. L., Monod, H., \& Bille, J. (1995). Mechanisms of resistance to azole antifungal agents in Candida albicans isolates from AIDS patients involve specific multidrug transporters. Antimicrobial Agents and Chemotherapy, 39, 2378-2386. https://doi.org/10.1128/aac.39.11.2378

Sanglard, D., Ischer, F., Koymans, L., \& Bille J. (1998). Amino acid substitutions in the cytochrome P-450 lanosterol 14alpha-demethylase (CYP51A1) from azole-resistant Candida albicans clinical isolates contribute to resistance to azole antifungal agents. Antimicrobial Agents and Chemotherapy, 42(2), 241-253. PMID: 9527767

Schaller, M., Borelli, C., Korting, H. C., \& Hube, B. (2005). Hydrolytic enzymes as virulence factors of Candida albicans. Mycoses, 48(6), 365-377. https://doi.org/10.1111/j.1439-0507.2005.01165.x

Serhan, G, Stack, C. M., Perrone, G. G., \& Morton, C. O. (2014). The polyene antifungals, amphotericin B and nystatin, cause cell death in Saccharomyces cerevisiae by a distinct mechanism to amphibian-derived antimicrobial peptides. Annals of Clinical Microbiology and Antimicrobials, 13(1), 18. https://doi.org/10.1186/1476-0711-13-18

Shapiro, R. S., Robbins, N., \& Cowen, L. E. (2011). Regulatory circuitry governing fungal development, drug resistance and disease. Microbiology and Molecular Biology Reviews, 75(2), 213-267. https://doi.org/10.1128/MMBR.00045-10

Silva, S., Negri, M., Henriques, M., Oliveira, R., Williams, D. W., \& Azeredo, J. (2012). Candida glabrata, Candida parapsilosis and Candida tropicalis: biology, epidemiology, pathogenicity and antifungal resistance. FEMS microbiology reviews, 36(2), 288-305. https://doi.org/10.1111/j.15746976.2011.00278.x

Silva, S. L., Lima, M. E. de, Santos, R. D. T. dos, \& Lima, E. de O. (2020). Onicomicoses por fungos do gênero Candida: uma revisão de literatura. Research, Society and Development, 9(8), e560985771. https://doi.org/10.33448/rsd-v9i8.5771

Sokol-Anderson, M. L., Brajtburg, J., \& Medoff, G. (1986). Amphotericin B-induced oxidative damage and killing of Candida albicans. Journal of Infectious Diseases, 154(1), 76-83. https://doi.org/10.1093/infdis/154.1.76

Sudbery, P. E. (2011). Growth of Candida albicans hyphae. Nature Reviews Microbiology, 9(10), 737-748. https://doi.org/10.1038/nrmicro2636 
Research, Society and Development, v. 10, n. 4, e43910414283, 2021

(CC BY 4.0) | ISSN 2525-3409 | DOI: http://dx.doi.org/10.33448/rsd-v10i4.14283

Thompson, D. S., Carlisle, P. L., \& Kadosh, D. (2011). Coevolution of morphology and virulence in Candida species. Eukaryotic cell, 10(9), 1173-1182. https://doi.org/10.1128/EC.05085-11

Vazquez, J. A., Arganoza, M. T., Boikov, D., Yoon, S., Sobel, J. D., \& Akins, R. A. (1998). Stable phenotypic resistance of Candida species to amphotericin B conferred by preexposure to subinhibitory levels of azoles. Journal of Clinical Microbiology, 36(9), 2690-2695. https://doi.org/10.1128/JCM.36.9.26902695.1998

Vázquez-González, D., Perusquía-Ortiz, A. M., Hundeiker, M., \& Bonifaz, A. (2013). Opportunistic yeast infections: candidiasis, cryptococcosis, trichosporonosis and geotrichosis. JDDG: Journal der Deutschen Dermatologischen Gesellschaft, 11(5), 381-394. https://doi.org/10.1111/ddg.12097

Vandeputte, P., Tronchin, G., Berges, T., Hennequin, C., Chabasse, D., \& Bouchara, J. P. (2007). Reduced susceptibility to polyenes associated with a missense mutation in the ERG6 gene in a clinical isolate of Candida glabrata with pseudohyphal growth. Antimicrobial Agents and Chemotherapy, 51(3), 982-990. https://doi.org/10.1128/AAC.01510-06

Vermes, A., Guchelaar, H.-J., \& Dankert, J. (2000) Flucytosine: a review of its pharmacology, clinical indications, pharmacokinetics, toxicity and drug interactions. Journal of Antimicrobial Chemotherapy, 46(2), 171-179. https://doi.org/10.1093/jac/46.2.171

Vincent, B. M., Lancaster, A. K., Scherz-Shouval, R., Whitesell, L., \& Lindquist S. (2013). Fitness trade-offs restrict the evolution of resistance to amphotericin B. PLoS Biology, 11(10), e1001692. https://doi.org/10.1371/journal.pbio.1001692

Wani, M. Y., Ahmad, A., Kumar, S., \& Sobral, A. J. (2017). Flucytosine analogues obtained through Biginelli reaction as efficient combinative antifungal agents. Microbial Pathogenesis, 105, 57-62. https://doi.org/10.1016/j.micpath.2017.02.006

Xie, J. L., Polvi, E. J., Shekhar-Guturja, T., \& Cowen, L. E. (2014). Elucidating drug resistance in human fungal pathogens. Future Microbiology, 9(4), 523542. https://doi.org/10.2217/fmb.14.18 